\title{
Kapitalisierung der Medienindustrie aus politökonomischer Perspektive
}

\author{
Manfred Knoche
}

Ansätze zur Kritik der politischen Ökonomie der gesellschaftlichen Kommunikation gebören in der Publizistik- und Kommunikationswissenschaft zu den „vergessenen Theorien". Aber angesichts des unübersebbaren Strukturwandels einer durch Deregulierung, Privatisierung, Digitalisierung, Konzentration, Globalisierung etc. „entfesselten" Medienindustrie erscheint es wissenschaftlich notwendig, die Entwicklung der Medienindustrie im engen Zusammenhang mit der ebenso unübersebbaren generellen Entwicklung eines „entfesselten“ Kapitalismus zu analysieren. In diesem Beitrag wird deshalb gezeigt, dass es die Analyse der Entwicklungsprozesse des Kapitalismus als dem zweifellos weltweit herrschenden Wirtschafts- und Gesellschaftssystem aus politökonomischer Perspektive ermöglicht, den Ökonomisierungs- bzw. Kommerzialisierungsprozess in der Medienindustrie hinsichtlich seiner Ursachen, Formen, Folgen und weiteren Entwicklung wissenschaftlich angemessen zu analysieren, erklären und teilweise zu prognostizieren. Theoretische Erklärungsansätze hierzu bieten die auf der Marx'schen Kritik der politischen Ökonomie basierenden Weiterentwicklungen einer aktuellen Kapitalismusanalyse und-kritik als historisch-materialistische Gesellschaftsanalyse. Dabei werden die dauerhaft grundlegenden Merkmale, Funktionsweisen und „Gesetzmäßigkeiten" kapitalistischer Produktionsweise und Gesellschaftsformation in Verbindung mit den Besonderheiten des aktuellen Kapitalisierungsprozesses in der Medienindustrie analysiert.

„Heute befindet sich der Kapitalismus meiner Auffassung nach zum erstenmal in einem Zustand, in dem die Kapitallogik genau so rein und unverfälscht funktioniert, wie Marx das im Kapital beschrieben hat.“

Oskar Negt 1997, 38

Was neuerdings verstärkt auch in der Publizistik- und Kommunikationswissenschaft als Ökonomisierung bzw. Kommerzialisierung der Medienindustrie thematisiert wird, ist aus politökonomischer Perspektive grundsätzlich ein altes Phänomen, das als ein wesentliches Strukturmerkmal privatwirtschaftlich organisierter Medienproduktion, -distribution und -konsumtion angesehen werden kann. Die Zugehörigkeit zum erwerbswirtschaftlich, also kommerziell organisierten Sektor der Privatwirtschaft kennzeichnet die Medien in den kapitalistisch organisierten Gesellschaften schon seit ihrem Aufkommen (vgl. Kiefer 1999, 705). Dennoch ist zu konstatieren, dass die etablierte deutschsprachige Publizistik- und Kommunikationswissenschaft erst am Ende des 20. Jahrhunderts plötzlich und eher verwundert festgestellt hat, dass die Massenmedien und damit auch die gesellschaftliche Kommunikation mehr und mehr ökonomisiert werden (vgl. Meier 1997, 173). Diese Verwunderung hätte weniger plötzlich oder zumindest früher sein können, wenn in dieser Wissenschaft nicht - ähnlich wie in allen anderen Wissenschaften - eine so weit verbreitete Enthaltsamkeit hinsichtlich der Rezeption und Anwendung der Marx'schen Kritik der politischen Ökonomie und ihrer aktuellen Weiterentwicklungen herrschte. Der derzeitig weltweit erkennbare, zum Teil neuartige (nicht neue) Ökonomisierungs- bzw. Kommerzialisierungsschub in der Medienindustrie eröffnet wissenschaftlich die Chance, dass dieses alte Phänomen aufgrund seiner neuartigen, besser sichtbaren und damit weniger bestreitbaren Formen von mehr Wis- 
senschaftlerInnen als bisher in seiner politisch-ökonomischen Grundlegung erkannt und darauf basierende wissenschaftliche Erkenntnisse anerkannt werden können.

\section{1. Ökonomisierung als Kapitalisierung der Medienindustrie}

Der Begriff der „Ökonomisierung“ greift aber in der Perspektive einer kritischen politischen Ökonomie zu kurz. Denn es geht vielmehr um eine weitere historische Phase der fortschreitenden „Kapitalisierung“ der privatwirtschaftlichen Medienindustrie ${ }^{1}$, d. h. um eine radikale Subsumtion des gesamten Mediensystems unter die allgemeinen Kapitalverwertungsbedingungen. Damit wird diese noch stärker als bisher in die spezifische kapitalistische Produktionsweise, das Verhältnis von Produktivkräften und Produktionsverhältnissen und die ökonomisch-politische Gesellschaftsformation (vgl. Altvater 1999) eingebunden, welche dem fortgeschrittenen Entwicklungsstand und den weiteren Bestandserfordernissen des Kapitalismus als dem weltweit herrschendem Wirtschaftsund Gesellschaftssystem entsprechen. Diese Kapitalisierung bedeutet vor allem: Medienproduktion wird noch umfassender als bisher in das gesamtwirtschaftliche System kapitalistischer Waren- und Mehrwertproduktion einbezogen. Sie ist damit auch intensiver als bisher den „Bewegungsgesetzen“ und „Zwängen“ von Produktion und Kapitalverwertung, von Profitmaximierung und Konkurrenz sowie von Akkumulation und Konzentration unterworfen. Gesamtgesellschaftlich relevant ist die damit zwangsläufig einhergehende weitere, zumeist als „Kommerzialisierung“ bezeichnete Kapitalisierung von Information, Bildung, Politik, Kultur, Unterhaltung sowie von Arbeits- und Lebensverhältnissen als Beitrag zur neoliberalen „Durchkapitalisierung“ aller Lebensbereiche (vgl. Röttger 1997, 18f.).

Es handelt sich um einen neuen Kapitalisierungsschub im dauerhaft fortschreitenden Kapitalisierungsprozess, der allerdings für die weitere Entwicklung der Medienindustrie auf der Basis der Privatisierung von ursprünglich nicht kapitalisierten Sektoren von grundsätzlicher Bedeutung ist. Wesentliche Kennzeichen dieses Kapitalisierungsschubs in der Medienindustrie sind

- eine Kapitalisierung über die Privatisierung, Deregulierung und Kommerzialisierung von zusätzlichen Sektoren der Medienindustrie, die bislang in Europa als staatlich oder öffentlich-rechtlich organisierte (Monopol-)Bereiche noch nicht der direkten (wohl aber der indirekten) Kapitalverwertung zugänglich waren (Hörfunk, Fernsehen, Telekommunikation, Internet);

- ein Strukturwandel der Medienindustrie, der sich vor allem in zunehmender Kommerzialisierung der Medieninhalteproduktion als Warenproduktion, in zunehmender internationaler ökonomischer und publizistischer Konzentration sowie in ökonomischen und institutionellen Verflechtungen (vgl. Knoche 1999a, 154ff.) traditioneller und neuer Mediensektoren miteinander und mit den übrigen Industrien zeigt (Medien als Infrastruktur, E-Commerce);

- eine intensivierte „Kapitalisierung“ des Verhältnisses von Staat und Medienwirtschaft sowie der staatlichen Medienpolitik als Medienwirtschaftspolitik;

- eine intensivierte „Kapitalisierung“ der ökonomischen und politischen gesamtwirtschaftlichen und -gesellschaftlichen Funktionserfüllung der Medienindustrie im weltweiten Strukturwandel (Transformationsprozess) des Kapitalismus.

1 Spezifische Probleme des ähnlich kommerzialisierten öffentlich-rechtlichen Rundfunks werden in diesem Beitrag nicht behandelt. 
Angesichts der unübersehbar umfangreichen - strukturell zum Teil neuartigen - „Entfesselung “ der Medienindustrie durch Deregulierung, Privatisierung, Kommerzialisierung, Globalisierung, Multimediatisierung, Medien- und Industrie-Konvergenz, Digitalisierung, Konzentration, Anonymisierung des Kapitals, E-Commerce etc. erscheint es mir als noch angemessener als bisher, die Entwicklung der Medienindustrie im engen Zusammenhang mit der ebenso unübersehbaren generellen Entwicklung eines „entfesselten“ Kapitalismus zu analysieren. Dies insbesondere deshalb, weil der gegenwärtige und höchstwahrscheinlich auch zukünftige Kapitalisierungsprozess in der Medienindustrie vor allem dadurch (neuartig) gekennzeichnet ist, dass es zu einer weit gehenden gegenseitigen Durchdringung von Medienindustrie und übriger Volkswirtschaft kommt.

\section{Kritik der politischen Ökonomie der Medien}

Die grundsätzliche Bedeutung der fortschreitenden Kapitalisierung der Medienindustrie im engen Zusammenhang mit der Entwicklung des kapitalistischen Wirtschafts- und Gesellschaftssystems weist auf die Notwendigkeit eines kritischen kapital- bzw. kapitalismuszentrierten Ansatzes (vgl. Knoche 1999a, Prokop 2000) in der Kommunikationswissenschaft hin, der wissenschaftlich als gegenstandsadäquat gelten kann. Solange jedoch in der Ost-West-System-Auseinandersetzung nach dem Zweiten Weltkrieg die Beschäftigung mit Kapitalismuskritik auf der Basis einer Kritik der politischen Ökonomie im Westen auch im Bereich der Wissenschaften zur „Marginalisierung“, schlechtesten Falls zum Berufsverbot führte ${ }^{2}$, gab es kein günstiges „Klima“ für die Entwicklung und Rezeption kritischer politökonomischer Ansätze zum lange schon erkennbaren gesellschaftlichen Problem der Ökonomisierung bzw. Kommerzialisierung der Medienindustrie.

Ansätze einer Kritik der politischen Ökonomie der gesellschaftlichen Kommunikation bzw. der Massenkommunikation ${ }^{3}$ wurden zwar vereinzelt im angelsächsischen Sprachraum in entsprechenden Arbeiten zur „Political Economy of Communication(s) (of the Media)“ vorgelegt (vgl. z. B. Mosco 1996, Golding/Murdock 1997, Sussman 1999, McChesney 2000). Auch in Deutschland wurden derartige Ansätze in den 70er Jahren kurzzeitig entwickelt (vgl. z. B. Berliner Autorenkollektiv Presse 1972, Dröge/Modelmog 1972, Holzer 1973, Hund 1976, Negt/Kluge 1972, Prokop 1974). Aber politische Ökonomie gesellschaftlicher Kommunikation gehört zweifellos zur „vergessenen Theorie“ (Robes 1990, vgl. Knoche 1999b, 76ff.) und gilt bis heute kaum als zitierfähig. Es gibt nur wenige Erinnerungs-, Wiederbelebungs- und aktuelle Anwendungsversuche für einen politökonomischen Ansatz in der deutschsprachigen Publizistik- und Kommunikationswissenschaft (vgl. Holzer 1994, Meier 1996/97 u. 1997, Knoche 1999a u. 1999b, Prokop 2000, Steininger 2000, 210ff.).

Der Gegenstandsbereich einer Kritik der politischen Ökonomie ist die kritische theoriegeleitete empirische Kapitalismusanalyse. Hierbei zeigt sich, dass diese politische

2 Es ist das Verdienst von Wolfgang R. Langenbucher, dass er jüngst im Gedenken an Horst Holzer mit deutlichen Worten auf die negativen Folgen einer „Hinwendung zum politökonomischen Denken“ in Deutschland hingewiesen hat (vgl. Langenbucher 2000).

3 Hierzu gibt es eine relative Vielzahl divergierender Ansätze in Nordamerika, Großbritannien und Deutschland. Zur Entwicklung und (gegenseitigen) Kritik der verschiedenen Ansätze vgl. Mosco 1996, $72 \mathrm{ff}$. 
Ökonomie nicht ein Zweig der Wirtschaftswissenschaft, sondern umfassende Gesellschaftswissenschaft ist (vgl. Kade 1977, Schikora 1992) ${ }^{4}$. Es geht um die Analyse und Kritik der gesellschaftlichen Voraussetzungen und Strukturbedingungen kapitalistischer Produktionsweise und damit um deren Funktionsweise und Dynamik (vgl. Conert 1997, 140). Anders ausgedrückt: Es geht um die Analyse und Kritik der „kapitalistischen Regulierung “ (Kisker 2000a, 66) der Produktions- und Lebensverhältnisse, d. h. des gesamten wirtschaftlichen, gesellschaftlichen, sozialen, politischen und kulturellen menschlichen Lebens. Kapitalismus wird dabei als historisch gewordene, grundsätzlich veränderbare Produktionsweise und Gesellschaftsform gesehen (vgl. Ganßmann, 1998, 23).

\section{Medienindustrie im Kapitalismus}

Für unseren Untersuchungsgegenstand ist also die Brauchbarkeit einer politischen Ökonomie als wissenschaftliche Theorie und Methode $\mathrm{zu}$ prüfen, die sich als auf der Marx'schen Kritik der politischen Ökonomie ${ }^{5}$ basierende Weiterentwicklung einer aktuellen Kapitalismusanalyse und -kritik als historisch-materialistische Gesellschaftsanalyse versteht. Dies wird seit längerem u. a. in Deutschland - auch auf der Basis umfangreicher Studien der internationalen wissenschaftlichen Literatur - von einigen WirtschaftswissenschaftlerInnen, SoziologInnen und PolitologInnen geleistet. Im Mittelpunkt des Interesses steht hierbei die kritische wissenschaftliche Auseinandersetzung vor allem mit

- dem aktuellen Transformationsprozess des Kapitalismus (vgl. Altvater/Haug/Negt u. a. 1999, Bischoff 1999, Hickel/Kisker/Mattfeldt/Troost 2000, Hirsch 1990), insbesondere mit dem Wandel der Rolle des Staates (vgl. Hirsch 1998, Kisker 2000b);

- dem Neoliberalismus (vgl. Bischoff/Deppe/Kisker 1998, Kisker 1998, Schui 2000), dem Keynesianismus, den Markt-Mythen und Wirtschaftskrisen (vgl. Zinn 1998);

- der aktuellen Wirtschaftspolitik (vgl. Hickel 1998, Huffschmid 1994);

- Konkurrenz und Konzentration (vgl. Bischoff/Boccara/Zinn u. a. 2000, Huffschmid 1994 u. 2000, Kisker 2000b) sowie der Globalisierung (vgl. Altvater/Mahnkopf 1999, Heinrich/Messner 1998, Kisker 2000a);

- der politischen Ökonomie der Finanzmärkte (vgl. Huffschmid 1999).

Aus politökonomischer Perspektive ${ }^{6}$ wird die aktuelle Kapitalisierung der privatwirtschaftlichen Medienindustrie im Rahmen dieser aktuellen Entwicklungstendenzen des gesamten kapitalistischen Wirtschafts- und Gesellschaftssystems betrachtet und erklärt.

4 Im Gegensatz zum Ansatz einer „Neuen Politischen Ökonomie“, der zwar auch gesellschaftliche und politische Zusammenhänge einbezieht, aber bewusst an der vorherrschenden neoklassischen Wirtschaftstheorie anknüpft und diese weiterentwickelt (vgl. Kiefer 2001, 53ff.).

5 Es geht also hier nicht um „Marxismus“ im politischen Sinn, sondern um eine kritische Anwendung und Weiterentwicklung von wissenschaftlichen Analyse- und Theorieansätzen von Karl Marx. Zur vielfältigen Literatur zur Kritik der politischen Ökonomie im 20. Jahrhundert vgl. Heinrich 1999, $196 \mathrm{ff}$.

6 Ziel dieses Beitrags ist es nicht, eine „geschlossene“ politisch-ökonomische Theorie der Medien zu präsentieren. Es werden nur einige Aspekte „aus politökonomischer Perspektive“ aufgezeigt, die mir in analoger Anwendung der umfangreichen, vielfältig empirisch belegten politökonomischen Analysen der von mir herangezogenen Wirtschafts- und Politikwissenschaftler in Verbindung mit den verfügbaren Kenntnissen zur Entwicklung der Medienindustrie als theoretisch fundiert sowie „empirisch belegt“ oder zumindest belegbar erscheinen. 
Aus der Sicht der hochkonzentrierten und international agierenden Privatwirtschaft war die Medienindustrie in Europa vor der Phase der europaweiten Privatisierungen und Deregulierungen ein unterentwickelter „Fremdkörper“, der sich in mehrfacher Hinsicht als hinderlich für die Kapitalverwertungs- und Expansionsinteressen erwies. Staatlich (Post- und Telekommunikation) oder öffentlich-rechtlich (Hörfunk und Fernsehen) organisierte Monopole und nicht-kapitalisierte Sektoren (Internet) waren nicht als Kapitalanlagesphäre zugänglich, ließen sich nur beschränkt im Rahmen infrastruktureller technisch-organisatorischer Rationalisierungsmaßnahmen nutzen, waren nur sehr eingeschränkt „kommerzialisierbar“ und erlaubten keine extensive Einbindung in „globale" weltweite Marketing-, Werbe- und PR-Strategien. Als hinderlich erwies sich hierbei auch der „Kapitalisierungs-Rückstand“ der traditionell privatwirtschaftlichen Mediensektoren der Presse- und Buchproduktion, deren „Nachteil“ bis heute in ihrer größtenteils mittelständischen und nationalen Gebundenheit gesehen wird. Im gesamtwirtschaftlichen Interesse wurde mit Hilfe weltweit einheitlicher neoliberaler Wirtschaftsund Gesellschaftspolitik der Nationalstaaten und der EU (Parolen: „Öffnung der Binnenmärkte“, „Internationale Wettbewerbsfähigkeit“) dieser „Nachholbedarf“ der Medienindustrie seit Mitte der 80er Jahre weitgehend gedeckt. Die politisch-ökonomische „Rückständigkeit“ der Medienindustrie in Europa war in ähnlicher Weise auch für die europäischen und vor allem die US-amerikanischen Medienkonzerne ein Hindernis für die Realisierung ihrer existenznotwendigen Expansionsstrategien. Auch für aufstrebende europäische Medienkonzerne gab es einen „Nachholbedarf“ hinsichtlich der Überwindung nationalstaatlicher Expansionsgrenzen durch Teilhabe an internationalen Fusionen, strategischen Allianzen und Unternehmens-Netzwerken der "global players“ (vgl. Knoche 2001).

Art, Umfang, Zeitpunkt und Verlauf der Kapitalisierung der Medienindustrie werden demnach von den dauerhaften ökonomischen Interessen und Grundproblemen der Medienindustrie in Verbindung mit gleichartigen Interessen und Grundproblemen der gesamten Wirtschaft bestimmt. Die ökonomischen und darauf bezogenen politischen Maßnahmen und mittelfristigen Strategien zur Problemlösung, allen voran des Problems der strukturellen Überakkumulation des Kapitals, führen allerdings regelmäßig zu neuen zyklischen „Krisen“ und langfristigen Folgeproblemen in Wirtschaft und Gesellschaft (Abbildung 1).

Die unter dem Druck der strukturellen Überakkumulation des Kapitals vorgenommene Reduzierung von Erweiterungsinvestitionen (sinkende Investitionsquoten) und stattdessen (vor allem technische) Rationalisierungsinvestitionen führen regelmäßig zum Abbau von Arbeitskräften (Arbeitslosigkeit), zur Intensivierung der Arbeit und zur Senkung bzw. Stagnation des Lohn- und Gehaltsniveaus. In Verbindung mit dem gleichzeitig forcierten Abbau des Sozialstaates führt dies zu sinkender Kaufkraft und entsprechendem Rückgang des Konsums. Dies führt zu verschärfter Konkurrenz zwischen den Unternehmen in weitgehend gesättigten bzw. stagnierenden Märkten und darüber zu zyklischen ökonomischen „Krisen“ des gesamten Wirtschaftssystems.

Die Kapitalisierung der Medienindustrie ist in diesem Kontext unter anderem deshalb von wachsender elementarer gesamtwirtschaftlicher und -gesellschaftlicher Bedeutung, weil sie auch eine wichtige Funktion in den notwendigen mittelfristigen Problemlösungsstrategien der gesamten Wirtschaft hat. Insofern ist es erklärbar, dass die Kapitalverwertungsinteressen (und deren dauerhafte grundsätzlich krisenhafte Gefährdung) in der gesamten Wirtschaft in Symbiose mit den spezifischen Kapitalverwertungsinteressen in der Medienindustrie den Kapitalisierungsprozess der Medienindustrie vorantreiben. 
Abbildung 1: Kapitalisierung der Medienindustrie als Problemlösungsstrategie für die gesamte Wirtschaft

\begin{tabular}{|c|c|c|c|}
\hline $\begin{array}{l}\text { Dauerhafte } \\
\text { Grundprobleme }\end{array}$ & $\begin{array}{l}\text { Mittelfristige } \\
\text { Problemlösungen }\end{array}$ & $\begin{array}{l}\text { Langfristige } \\
\text { Folgeprobleme }\end{array}$ & \\
\hline Maßnahmen & Strategien & Wirtschaft $\longleftarrow$ & $\begin{array}{c}\text { Gesellschaft } \\
\downarrow\end{array}$ \\
\hline Strukturelle & Kapitalisierung & Umverteilung & Umverteilung \\
\hline $\begin{array}{c}\text { Überakkumulation } \\
\downarrow\end{array}$ & Medienindustrie & Kapital/Profite & Güterkonsum \\
\hline Sinkende & Mediensektoren & Kapitalkonzentration & Massen- \\
\hline $\begin{array}{c}\text { Investitionsquoten } \\
\downarrow\end{array}$ & $\begin{array}{c}\text { als Kapitalanlage } \\
\downarrow\end{array}$ & $\begin{array}{c}\text { Kapitalvernichtung } \\
\downarrow\end{array}$ & arbeitslosigkeit \\
\hline Rationalisierungs- & Investitionen & Strukturelle & Abbau \\
\hline investitionen & Produktinnovationen & Überakkumulation & Sozialstaat \\
\hline$\downarrow$ & $\downarrow$ & $\downarrow$ & $\downarrow$ \\
\hline Abbau Arbeitskräfte & Umverteilung/ & Sinkende & Soziale \\
\hline $\begin{array}{c}\text { Intensivierung Arbeit } \\
\downarrow\end{array}$ & $\begin{array}{c}\text { Abbau Arbeitskräfte } \\
\downarrow\end{array}$ & $\begin{array}{c}\text { Profitraten } \\
\downarrow\end{array}$ & $\begin{array}{c}\text { Ungleichheiten } \\
\downarrow\end{array}$ \\
\hline Abbau & Staatliche & Verschärfung & Umwelt- \\
\hline Sozialstaat & Förderung & Konkurrenz & belastung \\
\hline$\downarrow$ & $\downarrow$ & .. $\downarrow$ & $\downarrow$ \\
\hline Sinkende Kaufkraft & Ausweitung & Ökonomische & Gesellschaftliche/ \\
\hline Rückgang Konsum & Werbung/PR & „Krise“ & politische „Krise“ \\
\hline Sinkende Profitraten & $\begin{array}{l}\qquad \\
\text { Förderung } \\
\text { „Konsumklima“ }\end{array}$ & & \\
\hline$\stackrel{\downarrow}{\downarrow}$ & $\downarrow$ & & \\
\hline Verschärfung & Förderung & & \\
\hline Konkurrenz & „Systemtreue“ & & \\
\hline Ökonomische & Umverteilung & & \\
\hline „Krise“ & $\begin{array}{l}\text { Konsumausgaben/ } \\
\text { Zeitbudgets }\end{array}$ & & \\
\hline
\end{tabular}

Die Interessen von (bislang) medienfremdem Kapital richten sich einerseits auf die Medienindustrie als neuer gewinnversprechender Anlagesphäre für überakkumuliertes, „überschüssiges“ Kapital. Andererseits zielen sie auf die Werbe-, PR- und Kapitalzirkulationsfunktion der Medien, deren intensive Nutzung von der gesamten Wirtschaft angesichts der Krisen der Überakkumulation, des Kaufkraftrückgangs und der damit verbundenen Konkurrenzverschärfung als eine notwendige und geeignete Problemlösungsstrategie angesehen wird.

Diese medienbezogenen Interessen und Strategien prinzipiell der gesamten Wirtschaft, allerdings hauptsächlich repräsentiert durch die Großunternehmen, sind mit ein Auslöser für Kapitalisierungsprozesse in der Medienindustrie. Denn sie bewegten - zusammen mit den Interessen von Medienunternehmen - die Regierungen in den verschiedenen europäischen Staaten dazu, im gesamtwirtschaftlichen Interesse durch gesetzliche Privatisierung und Deregulierung der Mediensektoren Hörfunk, Fernsehen, Telekommunikation und Internet Kapitalisierungs- und Kommerzialisierungs-Hindernisse zu beseitigen. Zum anderen wurde dadurch der Druck auf die in einem hohen Maße von Werbeeinnahmen abhängigen Medienunternehmen erhöht, ihre Werbe-, PRund Zirkulationsfunktion im Interesse der gesamten Wirtschaft und im existenznot- 
wendigen Eigeninteresse auf der Basis von Kapitalisierung und Kommerzialisierung der Medienproduktion noch effektiver als bislang zu erfüllen. In Verbindung damit wuchs auch die Notwendigkeit bzw. Bereitschaft von Medienunternehmen, ihren Kapitalbedarf verstärkt aus bislang medienfremdem Kapital bzw. durch einen Börsengang zu decken.

Das grundsätzliche Problem ist jedoch darin zu sehen, dass die Kapitalisierung der Medienindustrie - auch in ihrer Nutzung als mittelfristige Problemlösungsstrategie für die gesamte Wirtschaft - zwangsläufig langfristig zu erheblichen wirtschaftlichen und gesellschaftlichen Folgeproblemen führt (Abbildung 1). Aufgrund dessen, dass das handlungsleitende Strategieziel der Gewinnmaximierungsinteressen der Einzelkapitale als unumstrittenes Basisziel des Kapitalismus politisch legitimiert ist und durchgesetzt wird, kann es im ebenfalls politisch legitimierten Konkurrenzkampf der Einzelkapitale systematisch immer nur zu vorübergehenden einzelwirtschaftlichen Problemlösungen kommen, die in Wirklichkeit zu Problemverschärfungen für die Einzelkapitale und für die Gesamtwirtschaft führen. Denn es kommt auch über die Kapitalisierung der Medienindustrie zu einer Umverteilung von Kapital und Profit, die durch zunehmende Ungleichverteilungen gekennzeichnet ist: mit vorübergehenden „Gewinnern“ (Kapitalkonzentration) und (zum Teil „endgültigen“) „Verlierern“ (Kapitalvernichtung). Aber dies ist auch für die Gewinner keine dauerhafte Problemlösung, sondern eine Problemverschärfung, da die genannten dauerhaften Grundprobleme kapitalistischer Wirtschaft (strukturelle Überakkumulation, sinkende Profitraten, Verschärfung der Konkurrenz) auf höherem Niveau zwangsläufig zur nächsten ökonomischen „Krise“ führen.

Dies gilt in strukturell ähnlicher Weise auch für die langfristigen gesellschaftlichen Folgeprobleme, die durch die Kapitalisierung der Medienindustrie teilweise verstärkt werden. Es kommt in der Bevölkerung zu einer weit reichenden Umverteilung von Konsumgütern aller Art, die in Verbindung mit Lohn- und Gehaltseinbußen, Massenarbeitslosigkeit und Abbau des Sozialstaates zu einer Ausweitung der ökonomischen und sozialen Ungleichheiten führt. Dies verursacht einerseits, insbesondere aufgrund der damit verbundenen mangelnden Massenkaufkraft, die nächste ökonomische „Krise“ und letztendlich die nächste gesellschaftlich-politische „Krise“. Zur Lösung gesamtwirtschaftlicher und gesamtgesellschaftlicher Probleme, etwa im Sinne einer gesamtgesellschaftlichen Verteilungsgerechtigkeit, ist die Kapitalisierung der Medienindustrie folglich kaum ein geeignetes Mittel. Im Gegenteil: Sie fördert die weitere Kapitalisierung und Kommerzialisierung des gesamten sozialen und gesellschaftlichen Lebens mit den noch näher aufzuzeigenden negativen Folgen.

\section{Ursachen, Formen und Folgen der Kapitalisierung der Medienindustrie}

Das intensive Studium der generellen und aktuellen Entwicklungsprozesse des Kapitalismus ist meines Erachtens eine geeignete Grundlage, um den Kapitalisierungsprozess in der Medienindustrie hinsichtlich seiner Ursachen, Formen, Folgen und weiteren Entwicklung wissenschaftlich angemessen analysieren, erklären und teilweise prognostizieren zu können. Konkreter Ausgangspunkt ist die Beobachtung, dass die offensichtlich marktradikal „entfesselte“ Medienindustrie als integraler Funktionsbereich im Kapitalismus mit dem derzeitig erkennbaren, ebenfalls „entfesselten“ globalen Transformationsprozess des Kapitalismus zusammenhängt, der u. a. schlagwortartig als „Turbo“Kapitalismus (vgl. Altvater/Haug/Negt u. a. 1999) oder als „Shareholder“-Society (vgl. Deppe/Detje 1998, 171ff.) oder als „Kapitalismus pur“ (Bischoff/Deppe/Kisker 1998, 225) gekennzeichnet wird. 
M\&K 49. Jahrgang 2/2001

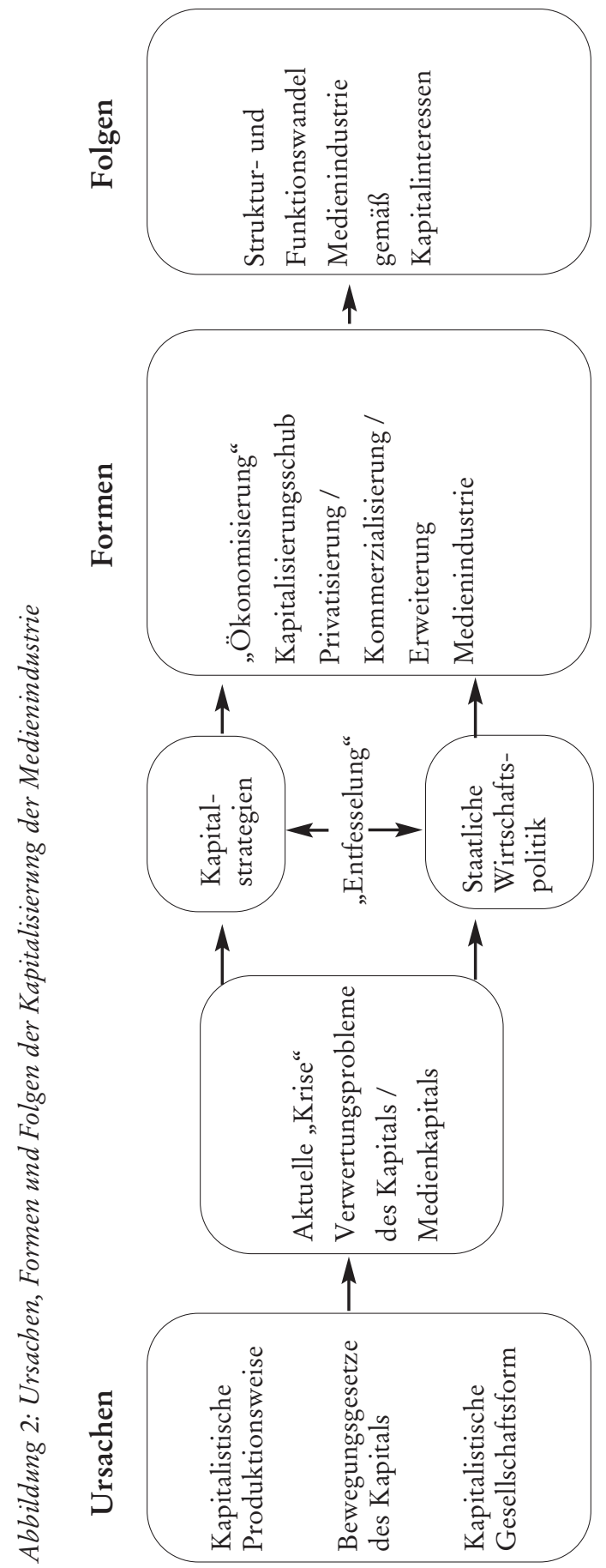


Aus kritischer politökonomischer Perspektive ist die aktuelle Kapitalisierung der Medienindustrie im engen Zusammenhang mit dem generellen Entwicklungsprozess des Kapitalismus mit den damit verbundenen Kapitalverwertungsproblemen in allen Wirtschaftszweigen zu sehen. Dabei gilt es jedoch auch, die ökonomisch-politischen Besonderbeiten der Medienproduktion, -distribution und -konsumtion im Vergleich zu anderen Wirtschaftsbereichen zu beachten. Sie ergeben sich vor allem durch die zusätzlichen (über die „normale“ ökonomische Kapitalverwertungsfunktion durch Produktion und Absatz der Medienprodukte hinausgehenden) gesamtwirtschaftlichen und gesamtgesellschaftlich-politischen Funktionen, die mit der privatwirtschaftlichen Medienproduktion in der Regel erfüllt werden (vgl. Holzer 1994, 195):

- einerseits die unverzichtbaren ökonomischen Funktionen für die gesamte Volkswirtschaft (allgemeine Werbe- und Warenzirkulationsfunktion, Kapitalverwertungsfunktion für Medientechnik, auch als Produktionsmittel),

- andererseits die ebenso unverzichtbaren ideologischen Funktionen für die Legitimation und Herrschaftssicherung des kapitalistischen Wirtschafts- und Gesellschaftssystems (Ideologieproduktionsfunktion) in der Bevölkerung,

- und schließlich die Funktionen für die Reproduktion der Arbeitskräfte durch Medienkonsum (Regenerationsfunktion).

Der Zusammenhang von Ursachen, Formen und Folgen der gegenwärtigen Kapitalisierung der Medienindustrie ist in Abbildung 2 zunächst im Überblick dargestellt. Als drei Ursachen-Komplexe eines zusammenwirkenden Ursachen-Bündels können unterschieden werden:

- die dauerhaft grundlegenden Wirkungsfaktoren kapitalistischer Produktionsweise und Gesellschaftsform sowie der Bewegungsgesetze des Kapitals (vgl. Altvater/ Hecker/Heinrich/Schaper-Rinkel 1999), denen die kapitalistische Medienindustrie im Grundsatz in gleicher Weise wie andere Industrien unterworfen ist;

- die Besonderbeiten der aktuellen „Krise“ mit den aktuellen Verwertungsproblemen des Kapitals bzw. des Medienkapitals, ursächlich mit dem gegenwärtig zu beobachtenden Entfesselungs- und Transformationsprozess des Kapitalismus verbunden;

- die konkreten aktuell wirksamen Kapital- bzw. Medienkapitalstrategien im Zusammenwirken mit den Entfesselungsleistungen staatlicher (Medien-)Wirtschaftspolitik (Privatisierung, Deregulierung, Konzentrationsförderung etc.) als Ursachen für Formen und Folgen des gegenwärtigen Kapitalisierungsschubs in der Medienindustrie (vgl. Knoche 1999a, 180ff.).

\subsection{Ursachen der Kapitalisierung}

In Abbildung 3 werden ausgewählte Ursachen der Kapitalisierung der Medienindustrie detaillierter dargestellt, als erstes die Faktoren, die grundsätzlich kennzeichnend für die kapitalistische Produktionsweise sind (vgl. u. a. Conert 1997, 141, Kisker 2000a, $66 \mathrm{ff}$.$) :$

- die spezifische Form des Kapitalverbältnisses: das rechtlich geschützte Privateigentum an den Produktionsmitteln sowie die daraus abgeleitete Verfügungsmacht über die abhängig Arbeitenden (Arbeitskraft als Ware) sowie das Recht der alleinigen Bestimmung der Produktionsziele und der Verwertung der produzierten Waren durch das Kapital,

- die spezifische Form kapitalistischer Produktionsverbältnisse als Herrschaftsverbältnisse von Kapital über Arbeit, 
- der spezifische widersprüchliche Zusammenhang von Produktivkräften (Verhältnis von konstantem Kapital = Produktionsmittel und Rohstoffe sowie variablem Kapital = Arbeitskraft) und Produktionsverbältnissen,

- die spezifische Form kapitalistischer Warenproduktion als Produktion von Werten (Gebrauchs- und Tauschwerte), wobei die Tauschwertrealisierung für die Kapitaleigner die Gebrauchswertinteressen der Konsumenten dominiert,

- die spezifische Form kapitalistischer Mebrwertproduktion durch Aneignung des durch Mehrarbeit der abhängig Arbeitenden produzierten Mehrwerts durch das Kapital,

- die spezifische Form des Zusammenhangs von Produktions-, Verwertungs- und Profitzwang mit Konkurrenz, Akkumulation, Konzentration und Zentralisation des Kapitals,

- die spezifische Form kapitalistischer Herrschaftssicherung durch das Zusammenwirken von Kapitaleignern und Staat (vgl. Nutzinger 1977, 222 ff.),

- die spezifische Form der Kapitalisierung der Gesellschaft über den Zusammenhang von Produktions- und Reproduktionsprozess der Menschen mit der Ungleichheit der Güter- und Einkommensverteilung und der damit verbundenen Ungleichheit der materiellen, politischen, kulturellen und sozialen Lebensverhältnisse.

Abbildung 3: Ursachen der Kapitalisierung der Medienindustrie

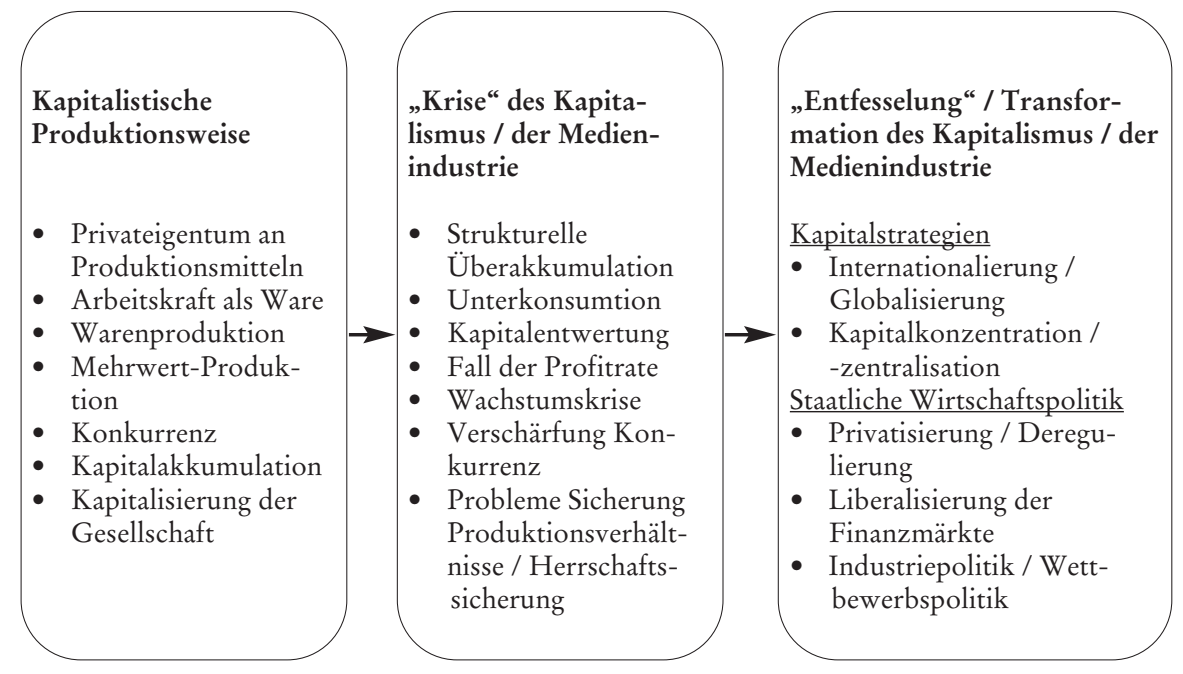

Berücksichtigt man diese grundlegenden Faktoren der kapitalistischen Produktionsweise, so wird erklärlich, weshalb alle Sektoren der privatwirtschaftlichen Medienindustrie in prinzipiell gleicher Weise einem ständig fortschreitenden Kapitalisierungsprozess unterworfen sind. Dies gilt für die Sektoren Presse, Buch, Film, Video, Musik, Radio, Fernsehen, für die im Kontext der Globalisierung der gesamten Wirtschaft und auch der Medienindustrie ein neuer Kapitalisierungsschub $\mathrm{zu}$ beobachten ist (vgl. Herman/McChesney 1997, 41ff.). Dieser zeigt sich zum Beispiel darin, dass einige Großun- 
ternehmen der - verglichen mit anderen Industrien ökonomisch bislang eher unbedeutenden - Medienindustrie in ihren Kapitalisierungsstrategien „Anschluss“ an das Kapitalisierungsniveau anderer Industrien gewinnen. So entwickelte sich zum Beispiel die Bertelsmann AG zum „Medienkonzern mit angeschlossener Investmentbank“ (Jakobs 2001, 110), d. h. der größte Teil des Gewinns wird seit Jahren nicht mit Medienproduktionen, sondern mit Börsendeals und dem Kauf/Verkauf von Unternehmen(-santeilen) erwirtschaftet.

Des Weiteren sind in Abbildung 3 die Faktoren genannt, die im Zusammenhang mit aktuellen Krisenerscheinungen des Kapitalismus und der Medienindustrie als relevante Ursachen für die weitere Kapitalisierung der Medienindustrie gelten können. Die Entwicklung des kapitalistischen Wirtschaftssystems ist generell in hohem Maße von struktureller Überakkumulation des Kapitals bestimmt, die als wesentlicher Auslöser für Wirtschaftskrisen auf der Basis einer disproportionierten Entwicklung (vgl. Zinn 1998, 23) wirkt. Disproportion bedeutet, dass grundsätzlich mehr produziert wird als abgesetzt, d.h. mit einer als angemessen angesehenen Profitrate verwertet werden kann, beziehungsweise dass aufgrund der Absatzprobleme weniger produziert wird, so dass es zu Überkapazitäten bei den Unternehmen kommt. Strukturelle Überakkumulation, die insbesondere durch Überinvestitionen, Überkapazitäten und Überproduktionen signalisiert wird, bedeutet, dass zu viel Kapital im Verhältnis zu den realisierbaren Profitraten akkumuliert wird, so dass es zur Gefahr einer Kapitalentwertung kommt. Das strategische Handeln der Unternehmen ist folglich darauf gerichtet, Maßnahmen zu ergreifen, um der mit der Überakkumulation einhergehenden Krisengefahr zu begegnen (vgl. Kisker 1998, 87ff.). Überakkumulation und Disproportionen sind Folgen der Konkurrenz zwischen den Unternehmen und den Branchen, die durch Überproduktion über den grundsätzlich durch mangelnde Kaufkraft beschränkten Bedarf bzw. die durch gesättigte oder nicht ausgeprägte Bedürfnisse beschränkte Nachfrage (Unterkonsumtion) hinausgehend im Konkurrenzkampf beim Verkauf ihrer Waren „gewinnen“ wollen.

Ursachen dieser die Kapitalverwertung elementar gefährdenden Wachstumsgrenzen sind Kaufkraftrückgänge infolge von Senkung des Lohn- und Gehaltsniveaus, Arbeitslosigkeit, Rückgang der Sozialleistungen, wachsender Sparzwang (Vorsorge, Versicherungen) sowie weitgehende Sättigung von „absoluten“, lebenswichtigen Bedürfnissen. Zur Überwindung dieser Wachstumsgrenzen und der damit verbundenen Gefahren der existenzgefährdenden Gewinnreduzierungen werden im Konkurrenzkampf von Unternehmen gleicher und verschiedener Branchen regelmäßig folgende Produktionsstrategien eingesetzt: (Teilweise) Verlagerung der Produktion von existenznotwendigen Gütern für die Befriedigung absoluter, lebenswichtiger Bedürfnisse hin zur Produkten für relative Bedürfnisse („Geltungs- bzw. Prestigekonsum“), damit verbunden (teilweise) Verlagerung der Produktion vom sekundären (industriellen) Sektor auf den tertiären (Dienstleistungs-)Sektor und (teilweise) Verlagerung der Produktion von materiellen zu immateriellen Gütern (vgl. Zinn 1998, 28ff.). Für die Anwendung derartiger kombinierter Unternehmensstrategien wird die Medienindustrie im Allgemeinen insofern als wachstumsträchtiges und Gewinn bringendes Geschäftsfeld angesehen, weil sie aufgrund des Zusammenspiels der drei Produktionsstrategien Wachstumschancen eröffnet.

In diesem Kontext ist der aktuelle Prozess der Kapitalisierung der Medienindustrie hauptsächlich in dreifacher Weise erklärbar. Die Kapitalisierung der Medienindustrie ist - erstens für traditionelle Medienunternehmen ein Mittel, ihre mit der Überakkumulation und der Konkurrenz verbundenen Kapitalverwertungsprobleme zu lösen, u. a. durch Anlage überschüssigen Kapitals in neuen privatisierten Mediensektoren bzw. in neuen Medienmärkten oder in Medienproduktinnovationen, 
- zweitens für Unternehmen aus anderen Industrien/Wirtschaftszweigen/Branchen ein Mittel, ihre Kapitalverwertungsprobleme durch eine verstärkte absatzfördernde Werbung und PR über die Medien zu lösen und

- drittens für Unternehmen aus anderen Industrien/Wirtschaftszweigen/Branchen ein Mittel, ihre Kapitalverwertungsprobleme durch Anlage ihres überschüssigen Kapitals in einer durch die Kapitalisierung erheblich erweiterten Medienindustrie zu lösen.

Schließlich wird in Abbildung 3 ein weiteres Ursachenbündel vorgestellt, das im Zusammenhang mit der „Entfesselung“ bzw. Transformation des Kapitalismus und der Medienindustrie relevant ist. Von „Entfesselung“ kann man z.B. insofern sprechen, als sich das Medienkapital wie das übrige Kapital durch Internationalisierung und Globalisierung von den „Fesseln“ der Nationalstaaten befreit. Eine ähnliche „Entfesselung“, nämlich die Befreiung von Kapitalverwertungshindernissen, wird insbesondere durch Privatisierung und Deregulierung von Rundfunk, Telekommunikation und Internet im Zusammenwirken mit staatlicher Wirtschaftspolitik erreicht. Im Rahmen staatlicher konzentrationsfördernder Industrie-, Standort- und Wettbewerbspolitik wird die Medienindustrie zusätzlich von Konzentrationshindernissen befreit (vgl. Knoche 1996). Schließlich befreit sich das Medienkapital im Rahmen neoliberaler Wirtschafts- und Gesellschaftspolitik von den „Fesseln“ des Sozialstaats und der parlamentarischen Demokratie. Das bedeutet zum Beispiel für die Medienindustrie, dass sie sich von den „Fesseln“ der Reste einer öffentlich-rechtlichen bzw. kulturellen Orientierung der Medienproduktion und der Medienpolitik befreit.

\subsection{Formen und Folgen der Kapitalisierung}

Als Formen des Kapitalisierungsschubs in der Medienindustrie können wir u.a. die in Abbildung 4 genannten Bereiche nennen, die in ihrer gegenwärtigen Form Ausdruck dieses Kapitalisierungsschubs sind, zusätzlich auch Produktdiversifikationen und -innovationen im Pressebereich. Man kann Formen dieses Kapitalisierungsschubs benennen, die für verschiedene Mediensektoren in gleicher Weise zu beobachten sind, z. B. die stärkere Einbeziehung der Medienproduktion in das gesamtwirtschaftliche System kapitalistischer Waren- und Mehrwertproduktion sowie in das System der Konsumwerbung. Damit verbunden ist ein intensiverer Einfluss von Produktions- und Kapitalverwertungszwang, von Profitmaximierungs- und Konkurrenzzwang sowie von Akkumulations- und Konzentrationszwang. Die prinzipielle Gleichartigkeit (zum Teil je nach Entwicklungsstand des Mediensektors ungleichzeitig) der Kapitalisierungsschübe in den einzelnen Mediensektoren ergibt sich aus der prinzipiellen Gleichartigkeit der aufgezeigten Ursachen hierfür, die sich wiederum aufgrund der Gleichartigkeit der Voraussetzungen - der privatwirtschaftlichen Organisationsform der Medien in einem kapitalistischen Wirtschafts- und Gesellschaftssystem - ergeben. Zusätzlich zeigt sich die Gleichartigkeit darin, dass durch technische und ökonomisch-institutionelle Konvergenz der Kapitalisierungsschub in einer Medienindustrie wirksam ist, die gerade durch den zunehmenden Abbau von Besonderheiten einzelner Mediensektoren gekennzeichnet ist.

Darüber hinausgehend kann man auf der Ebene einer differenzierteren Analyse für verschiedene Mediensektoren einer erweiterten Medien- und Kommunikationsindustrie auch einige spezifische Ursachen, Formen und Folgen dieses Kapitalisierungsschubs erkennen. Als bedeutsam erscheint mir hierzu die Unterscheidung (vgl. Knoche 1999a, 153ff.) nach: 
- Medienkapital, das zur Produktion bzw. Vervielfältigung von Programmen/Inhalten eingesetzt wird (die Mediensektoren Nachrichtenagentur, Presse, Buch, Hörfunk, Audio, Fernsehen, Film, Video, Online-Produktion), sowie

- medienbezogenem Kapital und Medieninfrastrukturkapital, das nur - und dies in der Regel nur teilweise - für die Produktion von medienbezogenen Produktions-, Komprimierungs-/Speicher-, Übertragungs-, Verschlüsselungs- und Empfangstechniken eingesetzt wird (Elektronikindustrie, Chemieindustrie, Computerindustrie, Telekommunikationsindustrie, Kabel- und Satellitenindustrie, Druckindustrie, Papierindustrie, Maschinenbauindustrie).

Die medienbezogenen Industriezweige haben schon seit längerem den im Kapitalismus „normalen“ fortschreitenden Kapitalisierungsprozess vollzogen (die Unternehmen dieser Industriezweige waren zum Beispiel auch schon vor der Privatisierung des Telekommunikationsbereichs Zulieferer von Medientechnik nach privatwirtschaftlichen Geschäftsgrundsätzen). Neuartiger, umfassender und politisch wesentlich bedeutsamer sind die Kapitalisierungsprozesse in den Mediensektoren, in denen die Kapitalverwertung mit programmlicher/inhaltlicher Produktion bzw. Vervielfältigung betrieben wird. Hier werden alle eingangs erwähnten grundlegenden Kapitalisierungsprozesse wirksam: von der Kapitalisierung über die Privatisierung, Deregulierung und Kommerzialisierung von Sektoren der Medienindustrie, die bislang in Europa staatlich oder öffentlichrechtlich, größtenteils als Monopole, organisiert waren, über die zunehmende Kommerzialisierung der Medieninhalteproduktion als Warenproduktion in allen Mediensektoren, den zunehmenden Einfluss der Werbewirtschaft und die Einbindung der Medien als Verkaufsmedien (Teleshopping, E-Commerce) bis hin zur gesamtwirtschaftlichen (Werbung, Absatzfunktion, Konsumklima), politischen (politisches Bewusstsein, Legitimation des kapitalistischen Wirtschafts- und Gesellschaftssystems) und gesellschaftlichen (Regeneration der Arbeitskräfte durch Unterhaltung) Funktionserfüllung der Medienproduktion im weltweiten Transformationsprozess des Kapitalismus.

Ein weiteres Unterscheidungsmerkmal zur Identifizierung tendenziell differenzierender Kapitalisierungsprozesse könnte die Finanzierungsart der Medienproduktion sein. Medienproduktionen, die ausschließlich oder überwiegend aus Werbung finanziert werden, so könnte man meinen, sind einem anderen Kapitalisierungsdruck ausgesetzt als Medienproduktionen, die ausschließlich über den Verkauf/Verleih finanziert werden. Diese Sichtweise verkennt jedoch, dass Medienproduktionen wie Film/Video und Audio (Musik) in weitem Maße auch so produziert werden, dass sie als werbegünstiges Umfeld bei Radio- und Fernsehsendungen dienen können. Die Kapitalisierung des Radio- und Fernsehsektors erfolgt nicht nur im Interesse der beteiligten Unternehmen und der Werbewirtschaft, sondern auch der Film- und Musikindustrie. Durch den Verkauf von Abspiel-Rechten an private Radio- und Fernsehunternehmen werden nicht nur zusätzliche Absatzmöglichkeiten geschaffen, sondern es wird auch das Problem mangelnder Kaufkraft und damit der Überproduktion/Überkapazitäten tendenziell verringert, da keine materiellen Produkte direkt einzeln an Konsumenten verkauft werden müssen wie beim CD- oder Video-Verkauf. Eine zusätzliche Variante der Kapitalisierung des Fernsehsektors, Pay-TV ohne Werbefinanzierung, dient der Lösung von Kapitalverwertungsproblemen, die sich aus Überkapazitäten an Filmen bzw. Filmrechten ergeben, für die der Absatz allein über werbefinanzierte Sender nicht ausreichend ist.

Schließlich lässt eine Unterscheidung in „traditionelle“ und „neue“ Mediensektoren Differenzierungen des Kapitalisierungsprozesses sichtbar werden. Während traditio- 
nelle Mediensektoren zum Teil schon seit langem auf einer hohen Stufe der Kapitalisierung angelangt sind (insbesondere Film- und Musikindustrie), ist die Kapitalisierung des Internet bzw. von Online-Medien erst in einer Anfangsphase. „Strategien für die digitale Wirtschaft" (European Communication Council Report 1999) gibt es zur Genüge, und vermutlich wird der Kapitalisierungsprozess der Online-Kommunikation schnell voranschreiten, da er auf der Basis eines hohen Kapitalisierungsgrades der gesamten interessierten Wirtschaft, darunter auch der Medienindustrie, vorangetrieben wird.

\section{Abbildung 4: Formen und Folgen der Kapitalisierung der Medienindustrie}

\section{Formen}

\section{Kapitalisierungsschub}

- Privatisierung Hörfunk/Fernsehen

- Privatisierung Telekommunikation

- Privatisierung Mobilkommunikation

- Privatisierung Internet

- Pay TV

- Digitalisierung

- Neue Werbeformen/Merchandising

- Produktdiversifikation/Produktinnovation

- Diversifizierte Multimedienunternehmen

- Börsengang/Kapitalbeschaffung

- Aktiengesellschaft

\section{Folgen}

Struktur-/Funktionswandel gemäß Kapitalinteressen

- Ausweitung Kommerzialisierung

- Massenkommunikation als Ware

- Kapitalkonzentration und -zentralisation

- Internationalisierung/Globalisierung

- Strukturelle Arbeitslosigkeit

- Prekäre Arbeitsverhältnisse

- Warenwerbung/Konsumklima

- Regeneration Bevölkerung

- Legitimation/Herrschaftssicherung

- Medienwirtschaftspolitik

Als allgemeinste Folge der weiteren Kapitalisierung der Medienindustrie ist zu beobachten, dass die Medienindustrie noch stärker als bisher dem allgemeinen und medienspezifischen Struktur- und Funktionswandel von Wirtschaft und Gesellschaft gemäß den Kapitalverwertungsinteressen unterzogen wird und diesen gleichzeitig mit beeinflusst. Die Folgen der damit einhergehenden Ausweitung der Kommerzialisierung der Medienproduktion erstrecken sich insbesondere auf

- die Gestaltung der Medienprodukte als Konsumgüter und als Waren in Konkurrenz zu anderen Waren,

- den Ausbau der Funktion der Medien als Werbe- bzw. Warenzirkulationsmittel für die gesamte Volkswirtschaft mit entsprechenden Folgen für die Inhalte der Medienprodukte,

- die Verstärkung internationaler Kapital- und Marktkonzentration sowie der Globalisierung der Medienindustrie,

- die Ausbreitung struktureller Arbeitslosigkeit und prekärer Arbeitsverhältnisse auch in der Medienindustrie,

- die Regeneration der Arbeitskräfte gemäß den Kapitalinteressen,

- die Beeinflussung der Bevölkerung hinsichtlich eines absatzfördernden „Konsumklimas" und eines politischen Bewusstseins gemäß den Kapitalinteressen,

- die weitere Ausrichtung staatlicher Medienpolitik an den Kapitalinteressen, 
- die weitere Legitimation und Herrschaftssicherung des internationalen kapitalistischen Wirtschafts- und Gesellschaftssystems, insbesondere in der gegenwärtig herrschenden Form des Neoliberalismus.

\section{Fazit}

Phänomene der Ökonomisierung und Kommerzialisierung der privatwirtschaftlichen Medienindustrie wurden in diesem Beitrag unter Anwendung von Ansätzen der Marx’schen Kritik der politischen Ökonomie und ihrer aktuellen Weiterentwicklungen als fortschreitende Kapitalisierung der Medienindustrie betrachtet. Damit wird ein weltweiter Prozess gekennzeichnet, im Verlauf dessen die Medienproduktion noch umfassender als bisher in das gesamtwirtschaftliche System kapitalistischer Waren- und Mehrwertproduktion einbezogen wird. Die damit verbundene Kapitalisierung von Information, Bildung, Politik, Kultur und Unterhaltung wird vor allem als Beitrag zur „Durchkapitalisierung“ aller Lebensbereiche im Zuge neoliberaler Wirtschafts- und Gesellschaftspolitik gesehen. Die Kapitalisierung der Medienindustrie folgt den Gesetzmäßigkeiten, die auch für andere Industriezweige wirksam sind, und sie erfüllt eine nicht unwesentliche Kapital- und Warenzirkulationsfunktion im Rahmen der Problemlösungsstrategien der gesamten Wirtschaft über ausgeprägte Werbe-, Marketing- und PRMaßnahmen. Besonderheiten der Kapitalisierung der Medienindustrie sind darin zu sehen, dass damit zusätzlich zu den ökonomischen Funktionen der Kapitalverwertung unverzichtbare politisch-ideologische Funktionen der Legitimierung des kapitalistischen Wirtschafts- und Gesellschaftssystems sowie Regenerationsfunktionen für die Bevölkerung erfüllt werden. Ursachen, Formen und Folgen der Kapitalisierung der Medienindustrie unterscheiden sich nicht prinzipiell von denjenigen, die in anderen Wirtschaftszweigen zu beobachten sind. Sie sind im Zusammenhang mit den allgemeinen Kapitalverwertungsproblemen und den Krisenphänomenen erklärbar, die dauerhaftes Kennzeichen kapitalistischer Wirtschaften sind. Es wird deutlich, dass die fortschreitende Kapitalisierung der Medienindustrie als irreversibler Prozess kaum ein geeignetes Mittel ist, demokratietheoretisch wünschbare Funktionserfüllungen der Medienproduktion zu fördern.

\section{Literatur}

Altvater, Elmar (1999): Kapital.doc, in: Elmar Altvater/Rolf Hecker/Michael Heinrich/Petra Schaper-Rinkel (Hrsg.): Kapital.doc: Das Kapital (Bd. 1) von Marx in Schaubildern mit Kommentaren. Münster, S. $12-187$.

Altvater, Elmar/Hecker, Rolf/Heinrich, Michael/Schaper-Rinkel, Petra (1999): Kapital.doc: Das Kapital (Bd. 1) von Marx in Schaubildern mit Kommentaren. Münster.

Altvater, Elmar/Mahnkopf, Birgit (1999): Grenzen der Globalisierung: Ökonomie, Ökologie und Politik in der Weltgesellschaft, 4. Aufl.. Münster.

Altvater, Elmar/Haug, Frigga/Negt, Oskar u. a. (1999): Turbo-Kapitalismus. Gesellschaft im Übergang ins 21. Jahrhundert. Hamburg.

Berliner Autorenkollektiv Presse (1972): Wie links können Journalisten sein? Pressefreiheit und Profit. Hamburg.

Bischoff, Joachim/Deppe, Frank/Kisker, Klaus Peter (Hrsg.) (1998): Das Ende des Neoliberalismus? Wie die Republik verändert wurde. Hamburg.

Bischoff, Joachim/Deppe, Frank/Kisker, Klaus Peter (1998): Licht am Ende des Tunnels?, in: Joachim Bischoff/Frank Deppe/Klaus Peter Kisker (Hrsg.): Das Ende des Neoliberalismus? Wie die Republik verändert wurde. Hamburg, S. 225 - 231. 
Bischoff, Joachim (1999): Der Kapitalismus des 21. Jahrhunderts. Systemkrise oder Rückkehr zur Prosperität? Hamburg.

Bischoff, Joachim/Boccara, Paul/Zinn, Karl Georg u. a. (2000): Die Fusions-Welle. Die Großkapitale und ihre ökonomische Macht. Hamburg.

Conert, Hansgeorg (1997): Zur Aktualität der Marx'schen Kapitalismuskritik, in: Zeitschrift Marxistische Erneuerung, 8, H. 31, S. $136-147$.

Deppe, Frank/Detje, Richard (1998): Globalisierung und die Folgen. Gewerkschaften unter falschen Sachzwängen, in: Joachim Bischoff/Frank Deppe/Klaus Peter Kisker (Hrsg.): Das Ende des Neoliberalismus? Wie die Republik verändert wurde. Hamburg, S. 154 $-176$.

Dröge, Franz/Modelmog, Ilse (1972): Wissen ohne Bewußtsein. Materialien zur Medienanalyse der Bundesrepublik Deutschland. Frankfurt a. M.

European Communication Council Report (1999): Die Internet-Ökonomie. Strategien für die digitale Wirtschaft. Berlin u. a.

Ganßmann, Heiner (1998): Soziologische Theorie im Anschluß an Marx, in: Michael Heinrich/ Dirk Messner (Hrsg.): Globalisierung und Perspektiven linker Politik. Festschrift für Elmar Altvater zum 60. Geburtstag. Münster, S. 22 - 36.

Golding, Peter/Murdock, Graham (Hrsg.) (1997): The Political Economy of the Media. Volume 1 +2 . Cheltenham/Brookfield.

Heinrich, Michael (1999): Kommentierte Literaturliste zur Kritik der politischen Ökonomie, in: Elmar Altvater/Rolf Hecker/Michael Heinrich/Petra Schaper-Rinkel (Hrsg.): Kapital.doc: Das Kapital (Bd. 1) von Marx in Schaubildern mit Kommentaren. Münster, S. 188 - 220.

Heinrich, Michael/Messner, Dirk (Hrsg.) (1998): Globalisierung und Perspektiven linker Politik. Festschrift für Elmar Altvater zum 60. Geburtstag. Münster.

Herman, Edward S./McChesney, Robert W. (1997): The Global Media. The New Missionaries of Corporate Capitalism. London/Washington.

Hickel, Rudolf (1998): Standort-Wahn und Euro-Angst. Die sieben Irrtümer der deutschen Wirtschaftspolitik. Hamburg.

Hickel, Rudolf/ Kisker, Klaus Peter/Mattfeldt, Harald/Troost, Axel (Hrsg.) (2000): Politik des Kapitals - heute. Festschrift zum 60. Geburtstag von Jörg Huffschmid. Hamburg.

Hirsch, Joachim (1990): Kapitalismus ohne Alternative? Materialistische Gesellschaftstheorie und Möglichkeiten einer sozialistischen Politik heute. Hamburg.

Hirsch, Joachim (1998): Vom Sicherheits- zum nationalen Wettbewerbsstaat. Berlin.

Holzer, Horst (1973): Kommunikationssoziologie. Reinbek.

Holzer, Horst (1994): Medienkommunikation. Einführung in handlungs- und gesellschaftstheoretische Konzeptionen. Opladen.

Huffschmid, Jörg (1994): Wem gehört Europa? Wirtschaftspolitik und Kapitalstrategien. Band 2: Kapitalstrategien in Europa. Heilbronn.

Huffschmid, Jörg (1999): Politische Ökonomie der Finanzmärkte. Hamburg.

Huffschmid, Jörg (2000): Megafusionen und „neue Ökonomie“, in: Joachim Bischoff/Paul Boccara/Georg Zinn u. a. (Hrsg.): Die Fusions-Welle. Die Großkapitale und ihre ökonomische Macht. Hamburg, S. 57 - 74.

Hund, Wulf D. (1976): Ware Nachricht und Informationsfetisch. Zur Theorie der gesellschaftlichen Kommunikation. Darmstadt.

Jakobs, Hans-Jürgen (2001): „Der Deal unseres Lebens“, in: Der Spiegel 7/2001, S. 110 - 112.

Kade, Gerhard (1977): Politische Ökonomie - heute, in: Winfried Vogt (Hrsg.): Seminar Politische Ökonomie. Zur Kritik der herrschenden Nationalökonomie, 2. Aufl.. Frankfurt a. M., S. 149 -168 .

Kiefer, Marie Luise (1999): Privatisierung und Kommerzialisierung der Medienwirtschaft als zeitgeschichtlicher Prozeß, in: Jürgen Wilke (Hrsg.): Massenmedien und Zeitgeschichte. Konstanz, S. $705-717$.

Kiefer, Marie Luise (2001): Medienökonomik. Einführung in eine ökonomische Theorie der Medien. München/Wien.

Kisker, Klaus Peter (1998): Der Neoliberalismus ist die Verschärfung, nicht die Lösung von Kri- 
sen, in: Joachim Bischoff/Frank Deppe/Klaus Peter Kisker (Hrsg.): Das Ende des Neoliberalismus? Wie die Republik verändert wurde. Hamburg, S. 81 - 91.

Kisker, Klaus Peter (2000a): Empörung der modernen Produktivkräfte gegen die modernen Produktionsverhältnisse im Zeitalter der »Globalisierung«, in: Rudolf Hickel/Klaus Peter Kisker/Harald Mattfeldt/Axel Troost (Hrsg.): Politik des Kapitals - heute. Festschrift zum 60. Geburtstag von Jörg Huffschmid. Hamburg, S. 65 - 73.

Kisker, Klaus Peter (2000b): Kapitalkonzentration und die Rolle des Staates im Zeitalter der Globalisierung, in: Joachim Bischoff/Paul Boccara/Karl Georg Zinn u. a. (Hrsg.): Die FusionsWelle. Die Großkapitale und ihre ökonomische Macht. Hamburg, S. 75 - 99.

Knoche, Manfred (1996): Konzentrationsförderung statt Konzentrationskontrolle. Die Konkordanz von Medienpolitik und Medienwirtschaft, in: Claudia Mast (Hrsg.): Markt - Macht - Medien. Publizistik im Spannungsfeld zwischen gesellschaftlicher Verantwortung und ökonomischen Zielen. Konstanz, S. $105-117$.

Knoche, Manfred (1999a): Das Kapital als Strukturwandler der Medienindustrie - und der Staat als sein Agent? Lehrstücke der Medienökonomie im Zeitalter digitaler Kommunikation, in: Manfred Knoche/Gabriele Siegert (Hrsg.): Strukturwandel der Medienwirtschaft im Zeitalter digitaler Kommunikation. München, S. 149 - 193.

Knoche, Manfred (1999b): Media Economics as a Subdiscipline of Communication Science, in: Hans-Bernd Brosius/Christina Holtz-Bacha (Hrsg.): The German communication yearbook. Cresskill/NJ, S. $69-100$.

Knoche, Manfred (2001): Die Folgen globaler Multimedien-Unternehmens-Allianzen, in: Uwe Hasebrink / Christiane Matzen (Hrsg.): Forschungsgegenstand Öffentliche Kommunikation. Funktionen, Aufgaben und Strukturen der Medienforschung. Baden-Baden (im Druck).

Langenbucher, Wolfgang R. (2000): Im Gedenken an Horst Holzer, in: Publizistik 45/4, S. 500 501.

McChesney, Robert W. (2000): The political economy of communication and the future of the field, in: Media, Culture \& Society, 22/1, S. 109 - 116.

Meier, Werner A. (1996/97): Globaler Medienwandel aus politökonomischer Perspektive, in: Medienwissenschaft Schweiz, 2/1996 + 1/1997, S. $70-85$.

Meier, Werner A. (1997): Zwischen traditioneller Medienökonomie und politischer Ökonomie gesellschaftlicher Kommunikation, in: Heinz Bonfadelli/Jürg Rathgeb (Hrsg.): Publizistikwissenschaftliche Basistheorien und ihre Praxistauglichkeit. Zürich, S. 173 - 183.

Mosco, Vincent (1996): The Political Economy of Communication. Rethinking and Renewal. London/Thousand Oaks.

Negt, Oskar (1997): Neuzugänge zum Marx’schen Denken, in: Zeitschrift Marxistische Erneuerung, 8, H. 30, S. $38-46$.

Negt, Oskar/Kluge, Alexander (1972): Öffentlichkeit und Erfahrung. Zur Organisationsanalyse von bürgerlicher und proletarischer Öffentlichkeit. Frankfurt a. M.

Nutzinger, Hans G. (1977): Wirtschaftstheorie aus der Sicht der Politischen Ökonomie, in: Winfried Vogt (Hrsg.): Seminar Politische Ökonomie. Zur Kritik der herrschenden Nationalökonomie, 2. Aufl.. Frankfurt a. M., S. $206-235$.

Prokop, Dieter (1974): Massenkultur und Spontaneität. Zur veränderten Warenform der Massenkommunikation im Spätkapitalismus. Frankfurt a. M.

Prokop, Dieter (2000): Der Medien-Kapitalismus. Das Lexikon der neuen kritischen Medienforschung. Hamburg.

Robes, Jochen (1990): Die vergessene Theorie. Historischer Materialismus und gesellschaftliche Kommunikation. Zur Rekonstruktion des theoretischen Gehalts und der historischen Entwicklung eines kommunikationswissenschaftlichen Ansatzes. Stuttgart.

Röttger, Bernd (1997): Neoliberale Globalisierung und eurokapitalistische Regulation. Die politische Konstitution des Marktes. Münster.

Schikora, Andreas (1992): Politische Ökonomie, in: Andreas Schikora/Angela Fiedler/Eckhard Hein (Hrsg.): Politische Ökonomie im Wandel. Festschrift für Klaus Peter Kisker. Marburg, S. $11-21$.

Schui, Herbert (2000): Neoliberalismus - Die Rechtfertigungslehre für die Konzentration von Ein- 
kommen und Vermögen, in: Herbert Schui/Eckart Spoo (Hrsg.): Geld ist genug da. Reichtum in Deutschland. 3. Aufl.. Heilbronn, S. $71-88$.

Steininger, Christian (2000): Zur politischen Ökonomie der Medien. Eine Untersuchung am Beispiel des dualen Rundfunksystems. Wien.

Sussman, Gerald (Hrsg.) (1999): Special Issue on the Political Economy of Communications, The Journal of Media Economics 12/2.

Zinn, Karl Georg (1998): Jenseits der Markt-Mythen. Wirtschaftskrisen: Ursachen und Auswege. Hamburg. 